\title{
Contribution of sonoelastography in the differentiation of benign and malignant breast masses: A comparative analysis on sonographic birads classification
}

Harun Arslan ${ }^{*}$, Zülküf Akdemir ${ }^{2}$, Alpaslan Yavuz ${ }^{1}$, Necat İslamoglu ${ }^{2}$, Sebahattin Çelik ${ }^{3}$, Mesut Özgökçe ${ }^{1}$, Abdussamet Batur ${ }^{1}$, Hüseyin Akdeniz ${ }^{1}$, Harun Egemen Tolunay ${ }^{4}$

${ }^{1}$ Department of Radiology, Van Yuzuncu Yil University, Dursun Odabaş Medical Center, Van, Turkey

${ }^{2}$ Department of Radiology, $V$ an Training and Research Hospital, Van, Turkey

${ }^{3}$ Department of General Surgery, Van Yuzuncu Yil University, Dursun Odabaş Medical Center, Van, Turkey

${ }^{4}$ Department of Obstetrics And Gynaecology, Van Yuzuncu Yil University, School of Medicine, Van, Turkey

\begin{abstract}
In this study, we aimed to investigate the effectivity of ultrasonographic elastography in the differentiation of benign and malignant lesions detected on B-mode ultrasonography.

The retrospective study included 133 consecutive patients who underwent B-mode USG followed by elastography between January 2013 and December 2015. The lesions were scored by using B-mode USG with breast imaging-report and data systems (BIRADS) and then sonoelastographic color-coded maps were obtained by elastography in a single session. Sonoelastographic scoring was achieved with a 5-point scoring system. Elasticity scores and strain ratio (SR) values were compared with histopathological findings. The 133 patients included $132(99.2 \%)$ women and $1(0.8 \%)$ man. Mean age was 40.45 \pm 14.8 years. The lesions were mostly localized in the left breast ( $\mathrm{n}=70,52.3 \%$ vs. $\mathrm{n}=63,47.7 \%)$. Depending on the pathological findings, $94(71.2 \%)$ lesions were classified as benign and $39(28.8 \%)$ as malignant. Mean SR value was $4.419 \pm 1.43$, with 5.5 in malignant lesions and 4.3 in benign lesions. At the cut-off value of 4.95 for SR, the sensitivity and specificity of USG in elastographic examination were $81.6 \%$ and $78.7 \%$, respectively. A significant correlation was found between the SR value and the BIRADS score calculated on USG (rho=0.86; two-tailed; $\mathrm{p}<0.001)$. Similarly, a significant correlation was found between the SR value and the elasticity score (rho=0.88; two-tailed; $\mathrm{p}<0.001$ ).

In conclusion, the use of sonoelastographic examination following the USG examination with B-mode ultrasonography can be a complementary diagnostic method that increases the specificity of USG. Moreover, it can also reduce unnecessary biopsies in lesions with suspicious malignancy.
\end{abstract}

Key Words: Breast cancer, elasticity imaging techniques, ultrasonography

\section{Introduction}

Mammography and ultrasonography (USG) are the gold-standard imaging methods used in the evaluation of breast masses. However, both methods are limited since mammography can lead to false-negative results in dense breasts and USG has low specificity. Sonoelastography is a USG-based technology used for the evaluation of tissue elasticity and characterization of lesions. Elastography resembles the conventional practice of examination with hand palpation and it is more sensitive and less subjective. Elasticity refers to the ability of tissue to return to its original shape and size after the removal of the external tension causing deformation of the tissue. Tissue deformation is inversely correlated with tissue stiffness. Adipose tissues undergo greater deformation when subjected to compression but return to their original shape and size quicker than fibrous and malignant tissues. Tissue elasticity is measured by the analysis of the echo signals acquired from the tissue during repetitive compressive movements of the transducer (1). The stiffness of the tissues investigated on strain elastography can be assigned different color-coded or gray scales based on their strain properties. In general, stiff tissues are coded in shades of blue, soft tissues in shades of red, and tissues with average stiffness in shades of green. Strain ratio (SR) is the ratio of the strain value of the tissues surrounding the investigated tissue to the strain value of the investigated tissue. The $\mathrm{SR}$ value is generally higher in stiff tissues since they undergo less compression and deformation compared to surrounding tissues. The SR value is accepted as a criterion for the comparison of the elasticity of different lesions $(2,3)$. Malignant tissues tend to be 100 times more elastic than normal tissues. Depending on this fact, the diagnostic compression technique has been developed. During compression, normal tissues become smaller, whereas malignant

*Corresponding Author: Harun Arslan MD., Van Yuzuncu Yil University, Dursun Odabaş1 Medical Center, Department of Radiology 65800 Tusba, Van/ Turkey Phone: +90 (532) 74688 35, E-mail: arslanharun.75@outlook.com

Received: 10.11.2017, Accepted: 21.11.2017 
Arslan et al / Contribution of sonoelastography in the differentiation of benign and malignant breast masses

tissues appear larger since they are relatively less elastic (4,5). Benign and malignant lesions can be differentiated by evaluating the contour features, size, color, strain ratio values, and the appearance of the lesions (5). In this study, we aimed to investigate the contribution of strain elastography to conventional USG in the differential diagnosis of benign and malignant breast masses.

\section{Material and methods}

The retrospective study included 133 consecutive patients who were admitted to Van District Training and Research Hospital Radiology Department and underwent B-mode USG followed by elastography between January 2013 and December 2015. The study protocol of the research was reviewed and confirmed by the local ethics committee. A written consent form was obtained from each patient. Sonography and elastography results were recorded for each patient. The sonography results were compared with those obtained by fine-needle aspiration (FNA) and tru-cut biopsy (TCB). B-mode USG and elastography were performed by using Toshiba Aplio 500 Ultrasound System (Toshiba Medical System) and conventional USG and elastography were performed by using a high-resolution linear 7-12 Mhz transducer. The lesions were evaluated on B-mode USG followed by elastography in a single session by a radiologist experienced in breast ultrasonography. All the radiological images were recorded on the hard disk of the USG device.

Radiological examination was performed prior to percutaneous biopsy and stereotactic biopsy. The patient was placed in the supine position prior to the sonoelastography procedure. Following the centralization of the lesion, rhythmic compression- decompression maneuvers were performed with the USG transducer perpendicular to the lesion, skin, and neck. Mild compression was applied to skin with the transducer to enable favorable compression for sonoelastographic images. Elastography was performed after the administration of B-mode USG.

The results obtained by both methods were evaluated both separately and together. The results obtained by conventional USG were classified based on the breast imaging-report and data system (BIRADS) (6,7). Accordingly, the lesions in category 2 were accepted as benign, category 3 as most probably benign, category 4 as lesions with suspicious abnormality, and category 5 as lesions with highly suspicious of malignancy. On elastography, "Strain ratio" and "Strain pattern" of the tissues were evaluated by using "Specific preset" settings. The scoring of strain pattern was conducted based on the Tsukuba scoring method developed by Itoh et al. (7). In this method, score 1 indicates completely green color-coded lesions with a soft and loose structure, score 2 indicates lesions coded in blue and green mosaic and their heterogeneously distributed soft-stiff internal structure, score 3 indicates lesions coded in blue in the center and green in the surrounding areas suggesting that the center of the lesion is harder and the outside is softer, score 4 indicates completely blue color-coded lesions suggesting that the entire structure is stiff and tight, and score 5 indicates blue encoding of a region that is larger than that of the size of the lesion, involving the lesion and its peripheral tissues, suggesting that the internal structure of the peripheral tissue is stiff due to the lesion and the desmoplastic reaction. Moreover, cystic lesions exhibit a unique "BGR" pattern of blue, green and red color-coding (Figure 1a, b).

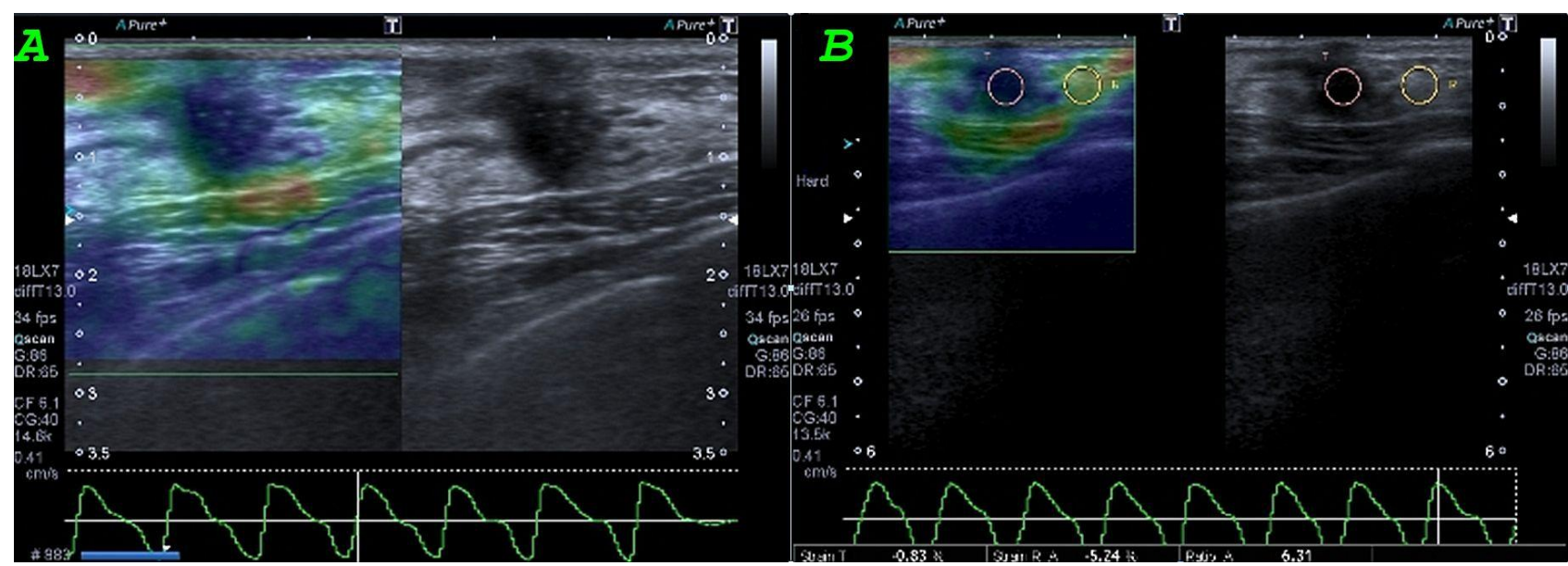

Fig. 1a). Invasive ductal carcinoma gray scale ultrasonography and elastography images b). Strain elastography images in invasive ductal carcinoma. 
The strain ratio was acquired as the numerical value calculated by the division of the strain value of the adipose tissue to the strain value of the lesion. Accordingly, the scores of 1,2 and 3 indicated benign lesions and the scores of 4 and 5 were accepted to indicate malignant lesions. Since the strain ratio is a quantitative method, a cut-off value was defined for this method (7).

Strain ratio (SR) value was calculated for each lesion. The SR value was accepted as the ratio of the elasticity value of the surrounding area to the elasticity value of the investigated tissue. During the calculation of the SR value, a region of interest (ROI) large enough to cover the area was placed on the area to be compared and the ratio of the reference ROI to the first ROI was obtained by measuring the ROI from the reference tissue that is on the same alignment. The size of the ROI cursor was adjusted according to the size of each lesion. However, the size of the ROI cursor has no standard reference value and has been shown to have no effect on the measurement results (8). The cursor was placed on the maximum areas of stiffness to obtain the highest SR value. Scoring of the sonoelastographic images and the measurement of the SR values were performed by a single radiologist blinded to the histopathologic diagnosis of the patients. The elasticity scores and the SR values were compared with histopathologic findings.

Statistical Analysis: Data were analyzed using SPSS 20.0 (IBM Corp., Armonk, NY, USA). Normal distribution of continuous variables (age and SR value) was analyzed by using visual methods (histograms and probability distribution charts) and a nonparametric test (Kolmogorov-Smirnov test).
Descriptive statistics were represented by mean and standard deviation (SD) for age, by median and minimum-maximum for the SR value, and by frequencies and percentages for the categorical and nominal variables. Differences between the SR values of benign and malignant lesions were compared by using Mann-Whitney $U$ test. The relation of BIRADS and elasticity scores with the differentiation of benign and malignant lesions was analyzed by using chisquare test, followed by Fischer's exact test as needed. The sensitivity and specificity of the SR value in the differentiation of benign and malignant lesions was analyzed by using receiver operating curve (ROC) analysis. Correlation between the SR value and the categorical variables was determined by Spearman's rank correlation coefficient. A $p$ value of $<0.05$ was accepted significant.

\section{Results}

The 133 patients included 132 (99.2\%) women and 1 $(0.8 \%)$ man. Mean age was $40.45 \pm 14.8$ years. The lesions were mostly localized in the left breast $(n=70$, $52.3 \%$ vs. $\mathrm{n}=63,47.7 \%$ ). Pathological results were as follows: fibroadenoma, $\mathrm{n}=63,47.7 \%$; invasive ductal carcinoma, $n=36,27.3 \%$; mastitis, $n=12,9.1 \%$; simple cyst, $\mathrm{n}=10,7.6 \%$; fibrosis, $\mathrm{n}=6,4.5 \%$; epithelial hyperplasia, $\mathrm{n}=3,2.3 \%$; invasive lobular carcinoma, $\mathrm{n}=1,0.8 \%$; medullary breast carcinoma, $\mathrm{n}=1,0.8 \%$. Depending on the pathological findings, $94(71.2 \%)$ lesions were classified as benign and $39(28.8 \%)$ as malignant. Table 1 presents the BIRADS scores, elastography scores, and SR values for benign and malignant lesions.

Table 1. BIRADS and Elastography Scores and Strain Ratio Values for Benign and Malignant Lesions

\begin{tabular}{|c|c|c|c|c|}
\hline & & Benign (n, $\%)$ & Malignant (n, \%) & $\mathrm{p}$ \\
\hline \multirow{5}{*}{ BIRADS } & 2 & $22.23 .4 \%$ & $0.0 .0 \%$ & \multirow{4}{*}{$<0.001^{*}$} \\
\hline & 3 & $42.44 .7 \%$ & $1.2 .6 \%$ & \\
\hline & 4 & $17.18 .1 \%$ & $4.10 .5 \%$ & \\
\hline & 5 & $13.13 .8 \%$ & $34.86 .8 \%$ & \\
\hline & Total & $94.100 \%$ & $38.100 \%$ & \multirow{7}{*}{$<0.001^{*}$} \\
\hline \multirow{6}{*}{ ELAST. } & 1 & $11.11 .7 \%$ & $0.0 .0 \%$ & \\
\hline & 2 & $11.11 .7 \%$ & $0.0 .0 \%$ & \\
\hline & 3 & $31.33 .0 \%$ & $1.2 .6 \%$ & \\
\hline & 4 & $21.22 .3 \%$ & $5.13 .2 \%$ & \\
\hline & 5 & $20.21 .3 \%$ & $33.84 .2 \%$ & \\
\hline & Total & $94.100 \%$ & $39.100 \%$ & \\
\hline \multicolumn{2}{|c|}{ Strain Ratio (median, min.-max) } & 4.3.(0.63-6.0) & 5.5.(4.4-7.0) & $<0.001 \pi$ \\
\hline
\end{tabular}

*Analyzed by Chi-Square test. " Analyzed by Mann-Whitney U test. 


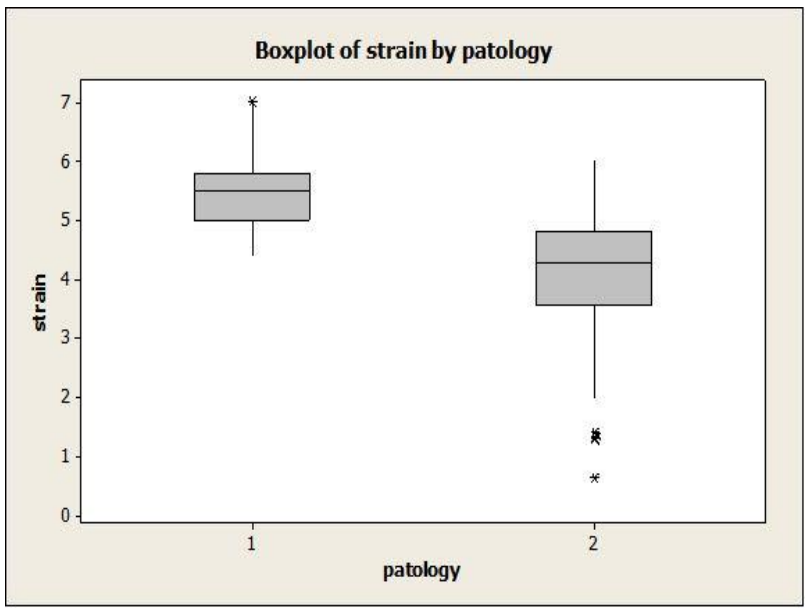

Fig. 2. Boxplot of strain elastography compared with pathology data.

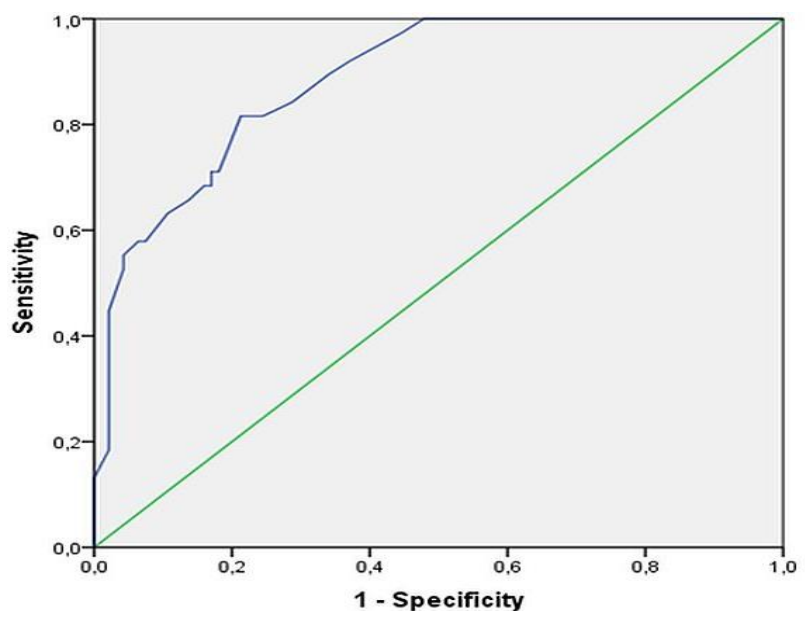

Fig. 3. ROC analysis of Sstrain Ratio in the differentiation of benign and malignant lesions $(\mathrm{rho}=0.885)$.

Table 2. BIRADS and Elastography Scores and Strain Ratio compared with sensitivity, specificity, PPV, NPV, accuracy of USG

\begin{tabular}{lccccc}
\hline & Sensitivity & Specificity & PPV & NPV & Accuracy \\
\hline BIRADS & $97.4 \%$ & $68.1 \%$ & $55.22 \%$ & $98.5 \%$ & $76.5 \%$ \\
ELAST. & $97.4 \%$ & $56.4 \%$ & $47.4 \%$ & $98.1 \%$ & $68.2 \%$ \\
Strain radio (cut-off value; 4.95) & $81.6 \%$ & $78.7 \%$ & $60.9 \%$ & $91.4 \%$ & $79.5 \%$ \\
\hline
\end{tabular}

Mean SR value was 4.419 \pm 1.43 , with 5.5 in malignant lesions and 4.3 in benign lesions (Figure 2). The cutoff value for SR value was accepted as 4.95 in ROC analysis (Figure 3). At this value, the sensitivity and specificity of USG in elastographic examination were $81.6 \%$ and $78.7 \%$, respectively (Table 2 ).

A significant correlation was found between the SR value and the BIRADS score calculated on USG (rho=0.86; two-tailed; $p<0.001$ ). Similarly, a significant correlation was found between the SR value and the elasticity score ( $\mathrm{rho}=0.88$; two-tailed; $p<0.001)$.

Table 2 presents the sensitivity, specificity, positive predictive value (PPV), negative predictive value (NPV), and accuracy rates of BIRADS, elastography, and the SI. As seen in the table, although the PPV of elastography was lower than that of BIRADS, the PPV of SR was higher than that of BIRADS and elastography. Similarly, the accuracy rate of the SR was higher than that of BIRADS and elastography. These findings indicate that the SR is an important diagnostic tool in patients presenting with a breast mass and an SR value of over 4.95 indicates a high risk of malignancy.

\section{Discussion}

Breast cancer in women is most commonly seen in patients aged 40-60 years in the world (9). Moreover, it is the most common type of cancer in women $(18 \%)$ and the lifetime risk of developing breast cancer is $7-10 \%$ (10). For these reasons, early diagnosis and treatment of breast cancer has been extensively studied in the literature. B-mode ultrasonography, breast masses can be evaluated based on their morphological features (11). Another recent development is a novel method known as elastography $(12,13)$. Differentiation of benign and malignant lesions can be performed by evaluating the contour features, size, color, strain ratio values, and the appearance of the lesions (14). Sonoelastographic evaluation of breast masses is often performed by using the Tsukuba scoring method developed by Itoh et al (7). In our study, we also used this method in the evaluation of our patients. The reported specificity and sensitivity of this method range between $70-99 \%$ and $35-97 \%$, respectively $(15,16)$. Similarly, the specificity and sensitivity of this method in our study were $78.7 \%$ and $81.6 \%$, respectively. These findings indicate that our study was consistent with most of the studies using this method in the evaluation of breast masses. Moreover, both our study and previous studies have shown that the use of sonoelastographic examination with a 5-point scoring system following the USG examination with B-mode ultrasonography can be a complementary diagnostic method for USG that increases the specificity of USG.

\footnotetext{
East J Med Volume:22, Number:4, October-December/2017
} 
Literature shows that BIRADS III and IV lesions are the most controversial lesions for the differential diagnosis of breast masses and most of these lesions are benign tumors. Moreover, the unnecessary biopsies performed for these lesions result in high medical costs around the world $(15,17)$. In such cases, the use of novel complementary methods following B-mode USG can be useful in the detection of benign lesions, thereby leading to reduction of unnecessary biopsies. Of these methods, strain elastography has been shown to be relatively superior in the detection of benign lesions $(15,17)$. Moreover, a strain ratio of over 3 has been shown to be indicative of suspicious malignancy $(15,16,18,19)$. However, there is no consensus on an ideal value or scoring system for the differentiation of benign and malignant lesions. Therefore, further studies with large patient series are needed. Gheonea et al. (20) evaluated 58 patients with breast masses and reported that the mean strain ratio was 2.08 in benign lesions and 6.28 in malignant lesions at a cut-off value of 3.67. In our study, mean strain ratio was 4.3 in benign lesions as opposed to 5.5 in malignant lesions. Moreover, a significant difference was found between the benign and malignant lesions in terms of elastography scores $(p=0.001)$. It was also revealed that the cut-off value (4.95) was highly sensitive and the strain ratio values established a perfect positive correlation with both BIRADS and elastography scores, which show that strain ratio can be a reliable method in the quantification of breast masses.

A recent study showed that BIRADS III lesions pose a low risk of malignancy and show a benign character within a 6-month follow-up period (21). The incidence of malignancy in BIRADS III and IV lesions has been shown to be $8 \%$ and $51.8 \%$, respectively, whereas malignancy has been reported in all BIRADS $\mathrm{V}$ lesions (22). In our study, the incidence of malignancy in BIRADS III and IV lesions was $2.3 \%$ and $19.1 \%$, respectively.

In the present study, we also investigated the effect of combined use of BIRADS classification and strain elastography on the accuracy of the histopathologic diagnosis. Of the 43 lesions classified as BIRADS III, $42(97.7 \%)$ lesions were histopathologically diagnosed as benign and the remaining $1(2.3 \%)$ lesion was diagnosed as invasive ductal carcinoma. However, in all the lesions, the elastography score was 3 or lower and the strain ratio was 4.5 or lower with a mean ratio of $1.44 \pm 0.52$. Moreover, the incidence of malignancy in these lesions was $2.3 \%$.

Of the 21 lesions classified as BIRADS IV, 17 (81\%) were diagnosed as benign and $4(19 \%)$ as malignant. The incidence of malignancy in these lesions was 19\%. The 17 benign lesions included 15 fibroadenomas and 2 fibrotic lesions, whereas all the 4 malignant lesions were invasive ductal carcinomas. In these lesions, the elastography scores were 4 or higher and the strain ratio values ranged between 4.5 and 6 .

Of the 47 lesions classified as BIRADS V, 32 lesions were histopathologically diagnosed as invasive ductal carcinoma, 11 as fibroadenoma, 2 as fibrotic lesion, 1 as medullary carcinoma, and 1 as lobular carcinoma. The strain ratio value was below 5.5 in 23 out of 47 lesions, of which 12 were histopathologically diagnosed as invasive ductal carcinoma, 11 as fibroadenoma, and 1 as medullary carcinoma. All the lesions with a strain ratio value of 4.4-7.0 had an elastography score of 5 .

Literature shows that the strain ratio can be erroneously measured below the cut-off value and the elastography scores can be erroneously measured higher than the actual scores in the presence of fibrosis and invasion in the peritumoral tissues (20). Moreover, 33 out of 53 lesions that were histopathologically diagnosed as malignant were found to be benign depending on the strain ratio values, which could be attributed to the presence of fibrosis and invasion in the peritumoral tissues. In addition, the strain ratio values of 23 out of 53 lesions were above the cut-off value. These findings suggest that color-coding may not be sufficient for proper differentiation of some lesions and thus combined use of elastography scores and strain ratio values in the evaluation of these lesions could yield more reliable results.

In our study, negative predictive value (NPV) was 10 and the number of false-negative patients was 17 at the cut-off value of 4.95. On the other hand, the positive predictive value (PPV) of BIRADS classification was $60.1 \%$ and the NPV was $91 \%$ (Table 2). We consider that these findings could be associated with bleeding malignant lesions, cystic lesions, and the tumors with a high risk of necrosis such as medullary carcinoma (23). Elastographic measurements have been shown to be affected by a number of factors including breast edema and the morphological changes caused by previous breast surgeries such as scarring and fibrosis (20). Moreover, presence of calcification, organization, abundant stromal cells, and excess fibrosis in the lesion has been shown to decrease tissue elasticity, resulting in false-positive results $(20,23-25)$.

Our study has several limitations. The primary limitation is that we did not use shear-wave elastography which is a novel method that might have yielded better quantitative results. Another limitation is that the strain elastography is operator-dependent and we used a limited number of methods for 
Arslan et al / Contribution of sonoelastography in the differentiation of benign and malignant breast masses

histopathological diagnosis. The final limitation is that the sonoelastographic examination was not independent of B-mode USG examination, mainly because sonoelastography is performed with colorcoded maps that are superimposed on B-mode USG images.

In this study, unlike the previous studies, we investigated the correlation between strain elastography and BIRADS classification system. The results showed a significant correlation between BIRADS classification system and strain elastography. It was also revealed that the strain ratio values increased as the BIRADS score increased, which shows that BIRADS classification system could be a useful method for the differentiation of BIRADS III and IV lesions, thereby leading to reduction of unnecessary biopsies.

In our study, although we had low strain ratio values in some of the malignant lesions, we had high elastography scores in the surrounding tissues. We consider that this situation was caused by the presence of peritumoral invasion or fibrosis in peritumoral tissues. In addition, we also had a high elastography score in the surrounding parenchyma of a benign lesion due to the same reasons. These issues were mentioned in the limitations of our study. We believe that further studies are needed to substantiate our findings.

In conclusion, with this method, unnecessary biopsies can be reduced in lesions with suspicious malignancy. Therefore, we believe that the clinical use of ultrasonographic elastography should be promoted.

Conflict of Interest: The authors declare that they have no conflict of interest

\section{References}

1. Garra BS. Imaging and estimation of tissue elasticity by ultrasound. Ultrasound Q 2007; 23: 255-268.

2. Bamber J, Cosgrove D, Dietrich CF, et al. F. EFSUMB guidelines and recommendations on the clinical use of ultrasound elastography. Part 1: Basic principles and technology. Ultraschall Med 2013; 34 : 169-184.

3. Erdoğan S. Meme Kitlelerinin Değerlendirilmesinde Nükleer Tip Yaklaşimi. Cerrahpaşa Tip Dergisi 2003; 34.

4. Yamamoto A, Fukushima H, Okamura R, et al. K. Dynamic helical CT mammography of breast cancer. Radiat Med 2006; 24: 35-40.

5. Zhao QL, Ruan LT, Zhang H, Yin YM, Duan SX. Diagnosis of solid breast lesions by elastography 5point score and strain ratio method. Eur J Radiol 2012; 81: 3245-3249.
6. Radiology ACo. Breast imaging reporting and data system. BI-RADS 2003.

7. Itoh $\mathrm{A}$, Ueno $\mathrm{E}$, Tohno $\mathrm{E}$, et al. Breast disease: clinical application of US elastography for diagnosis. Radiology 2006; 239: 341-350.

8. Balleyguier C, Canale S, Ben Hassen W, et al. Breast elasticity: principles, technique, results: an update and overview of commercially available software. Eur J Radiol 2013; 82: 427-434.

9. Greenlee RT, Murray T, Bolden S, Wingo PA. Cancer statistics, 2000. CA Cancer J Clin 2000; 50: 7-33.

10. Haydaroğlu A, Dubova S, Özsaran Z, et al. Breast cancer in Ege University "evaluation of 3897 cases". Eur J Breast Health 2005; 1: 6-11.

11. Stachs A, Hartmann S, Stubert J, et al. Differentiating between malignant and benign breast masses: factors limiting sonoelastographic strain ratio. Ultraschall Med 2013; 34: 131-136.

12. Ophir J, Cespedes I, Ponnekanti H, Yazdi Y, Li X. Elastography: a quantitative method for imaging the elasticity of biological tissues. Ultrason Imaging 1991; 13: 111-134.

13. Garra BS, Cespedes EI, Ophir J, et all. Elastography of breast lesions: initial clinical results. Radiology 1997; 202:79-86.

14. Zhi H, Xiao XY, Yang HY, Ou B, Wen YL, Luo BM. Ultrasonic elastography in breast cancer diagnosis: strain ratio vs 5-point scale. Acad Radiol 2010; 17: 1227-1233.

15. Sadigh G, Carlos RC, Neal CH, Dwamena BA. Ultrasonographic differentiation of malignant from benign breast lesions: a meta-analytic comparison of elasticity and BIRADS scoring. Breast Cancer Res Treat 2012; 133: 23-35.

16. Yerli H, Yilmaz T, Ural B, Gulay H. The diagnostic importance of evaluation of solid breast masses by sonoelastography. Ulus Cerrahi Derg 2013; 29: 6771.

17. Gazioğlu D, Büyükaşık O, Hasdemir AO, Kargıcı H. BIRADS 3 ve 4 Meme Lezyonlarına Yaklaşım: Hangi Olgulara Biyopsi Yapılmalı? Turgut Özal Tip Merkezi Dergisi 2009; 16.

18. Cho EY, Ko ES, Han BK, et al. Shear-wave elastography in invasive ductal carcinoma: correlation between quantitative maximum elasticity value and detailed pathological findings. Acta Radiol 2016; 57: 521-528.

19. Ganau S, Andreu FJ, Escribano F, et al.Shear-wave elastography and immunohistochemical profiles in invasive breast cancer: evaluation of maximum and mean elasticity values. Eur J Radiol 2015; 84: 617622.

20. Gheonea IA, Stoica Z, Bondari S. Differential diagnosis of breast lesions using ultrasound elastography. Indian J Radiol Imaging 2011; 21: 301-305. 
21. Kerlikowske K, Smith-Bindman R, Ljung BM, Grady D. Evaluation of abnormal mammography results and palpable breast abnormalities. Ann Intern Med 2003; 139: 274-284.

22. Abuoglu HH, Gunay E, Sunamak O, Yigitbasi MR. Diagnostic Value of Frozen Section in Patients with Non-Palpable Breast Lesions. Chirurgia (Bucur) 2016; 111: 500-504.

23. Mutala TM, Ndaiga P, Aywak A. Comparison of qualitative and semiquantitative strain elastography in breast lesions for diagnostic accuracy. Cancer Imaging 2016; 16: 12.

24. Thomas A, Degenhardt F, Farrokh A, Wojcinski S, Slowinski T, Fischer T. Significant differentiation of focal breast lesions: calculation of strain ratio in breast sonoelastography. Acad Radiol 2010; 17: 558563.

25. Sigrist RMS, Liau J, Kaffas AE, Chammas MC, Willmann JK. Ultrasound Elastography: Review of Techniques and Clinical Applications. Theranostics 2017; 7: 1303-1329. 